\title{
Numerical Simulation of the Fractures of Anisotropic Materials Characterized by the High Degree of Anisotropy of Elongation at Break
}

\author{
S. V. Kobenko ${ }^{1, \text { a) }}$, M. N. Krivosheina ${ }^{2, b)}$, M. A. Kozlova ${ }^{2, c)}$, \\ E. V. Tuch ${ }^{2, \text { d) }}$, and I. Yu. Konysheva ${ }^{3, \text { e) }}$ \\ ${ }^{1}$ Nizhnevartovsk State University, Nizhnevartovsk, 628605, Russia \\ ${ }^{2}$ Institute of Strength Physics and Materials Science SB RAS, Tomsk, 634055, Russia \\ ${ }^{3}$ National Research Tomsk Polytechnic University, Tomsk, 634050, Russia \\ a) Corresponding author: sergeyvk@inbox.ru \\ b) marina_nkr@mail.ru \\ c) kozlovama@mail.ru \\ d) elenatuch@yandex.ru \\ e) irishkak@mail.ru
}

\begin{abstract}
The failure criteria of anisotropic materials, the criteria being expressed via deformations, are rarely applied in practice, and are usually used for low-ductility materials. Another situation involves the simulation of failures in materials with the high anisotropy of mechanical properties and that suffer elastic-brittle fracture. For such materials the failure criteria are formulated using ultimate strains and strain tensor invariants. For simulating the fractures of materials that are characterized by the anisotropy of strength properties, the failure criteria are applied with the critical values of stresses and strains, as well as their intensities. Using the value of cumulative plastic strain as a failure criterion does reflect the anisotropy of elastic and plastic properties of a material, but it does not reflect that of the strength properties. The application of this method allows accounting the total plastic strain induced by tension and compression in the conditions of the wave strain of targets during their impact loading.
\end{abstract}

Keywords: impact loading, plastic deformation, numerical simulation, failure criterion

\section{INTRODUCTION}

This paper describes the application of a new criterion of anisotropic media failure formulated via the accumulated plastic strain. This criterion simulates the fracture of targets made from anisotropic 2024 aluminum alloy during the dynamic loading in $3 \mathrm{D}$ formulation. It features the possibility of analyzing the ultimate strain contribution in different directions, and ultimate shear strain in the material symmetry planes into the resulting fracture pattern. This criterion allows simulating the process of crater formation and shear plugging of the target [1].

The article reveals the contribution of the anisotropy of target material fracture characteristics using the failure criterion, which represents 6 different accumulated plastic strains for momentless orthotropic materials [2]. The paper contains the analysis of the fracture of target made from anisotropic 2024 aluminum alloy loaded by a steel projectile within the velocity range from 200 to $600 \mathrm{~m} / \mathrm{s}$.

\section{STATEMENT OF THE PROBLEM}

All calculations were performed using the finite elements method in 3D formulation using the proprietary software. The volumetric configurations of the projectile $\left(D_{1}\right)$ and the target $\left(D_{2}\right)$ were represented tetrahedrons (see Fig. 1).

International Conference on Physical Mesomechanics of Multilevel Systems 2014

AIP Conf. Proc. 1623, 267-270 (2014); doi: 10.1063/1.4898933

(C) 2014 AIP Publishing LLC 978-0-7354-1260-6/\$30.00 


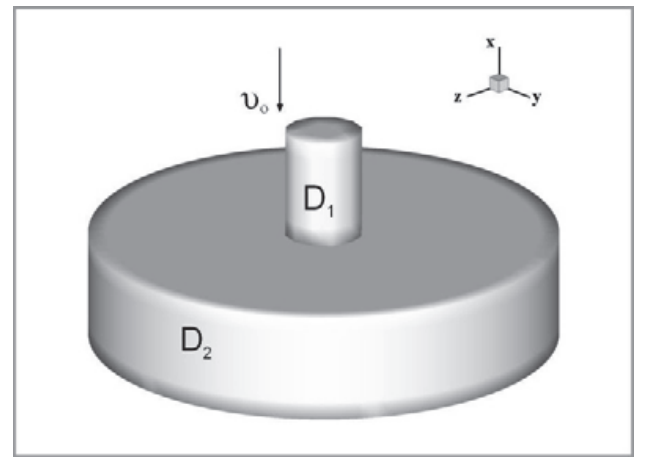

FIGURE 1. Initial volumetric configuration of the projectile and the target

\section{SIMULATION OF ELASTOPLASTIC DEFORMATION OF ORTHOTROPIC MATERIAL}

A set of equations describing the non-stationary adiabatic motion of a compressive anisotropic medium includes the following [3]:

equation of continuity:

$$
\frac{\partial \rho}{\partial t}+\operatorname{div} \rho \bar{v}=0
$$

equation of continuous medium motion:

$$
\rho \frac{\mathrm{d} v^{k}}{\mathrm{~d} t}=\frac{\partial \sigma^{k i}}{\partial x_{i}}+F^{k}
$$

and energy equation:

$$
\frac{\mathrm{d} E}{\mathrm{~d} t}=\frac{1}{\rho} \sigma^{i j} e_{i j}
$$

where $\rho$ is medium density, $\bar{v}$ is velocity vector, $F^{k}$ are the components of the vector of mass forces, $\sigma_{i j}$ are the contravariant components of symmetric stress tensor and $E$ is specific internal energy.

$$
e_{i j}=\frac{1}{2}\left(\nabla_{i} v_{j}+\nabla_{j} v_{i}\right)
$$

where $e_{i j}$ are the components of straining rate symmetric tensor, $v_{i}$ are velocity vector components, while $i, j=1$, 2,3 .

Let us assume that the ultimate strain can be formulated as a sum of elastic and plastic strains, the plastic flow of material is unaffected by the hydrostatic pressure (such assumption is valid in case of the elastic and plastic properties of a material having the low degree of anisotropy) and the elastic properties of a material are unaffected by the plastic strain.

Elastic behavior of a material is described by the Hook's generalized law:

$$
\frac{\mathrm{d} \sigma_{i j}}{\mathrm{~d} t}=C_{i j k l} e_{k l},
$$

where $C_{i j k l}$ are the components of the tensor of elastic constants.

By calculating plastic strain, the total stress tensor is as follows:

$$
\sigma^{k i}=-P \delta_{k i}+S^{k i}
$$

where $P$ is hydrostatic pressure, $S^{k i}$ is the deviator part of stress tensor and $\delta_{k i}$ is Kronecker symbol. 


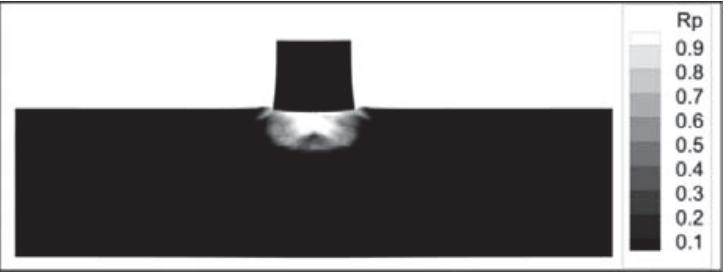

(a)

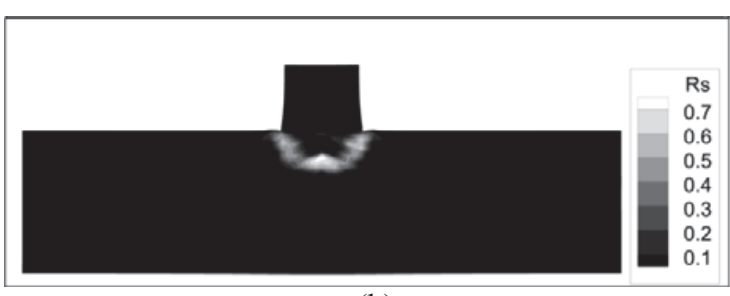

(b)

FIGURE 2. Material fracture zone during the compression (a) and tension (b) in the target material at the moment of $40 \mu \mathrm{s}$ $\left(V_{0}=200 \mathrm{~m} / \mathrm{s}\right)$. Considering only one ultimate strain

The pressure in material was estimated according to Mie-Gruneisen EOS as the function of specific internal energy $E$ and density $\rho$ :

$$
P=\sum_{n=1}^{3} K_{n}\left(\frac{V}{V_{0}}-1\right)^{n}\left[1-K_{0}\left(\frac{V_{0}}{V}-1\right) / 2\right]+K_{0} \rho E,
$$

where $K_{0}$ is Gruneisen coefficient, $K_{1}, K_{2}$ and $K_{3}$ are material constants, and $V_{0}$ is initial specific volume.

Let us assume the following formulation of associated flow rule:

$$
\mathrm{d} \varepsilon_{i j}^{\mathrm{p}}=\mathrm{d} \lambda \frac{\partial f}{\partial \sigma_{i j}},
$$

where $\mathrm{d} \lambda=0$ in the case of elastic strain, is positive for the plastic deformation and is determined via the plasticity condition, $\mathrm{d} \varepsilon^{ð}$ is plastic strain increment and $f$ is plasticity function.

$$
\varepsilon_{i}^{\mathrm{p}}=\int \mathrm{d} \varepsilon_{i}^{\mathrm{p}}, i=1, \ldots, 6 .
$$

The criterion of the ultimate plastic strain (9) was varied to identify its effect on the final proportion of the shearinduced fractured material.

The stresses in the element rigidly turned in space are recalculated using the Yaumann's derivative and are restricted by the reference frame:

$$
\frac{\mathrm{D} \sigma^{i j}}{\mathrm{D} t}=\frac{\mathrm{d} \sigma^{i j}}{\mathrm{~d} t}-\sigma^{i k} \omega_{j k}-\sigma^{j k} \omega_{i k}
$$

where $\omega_{i j}=\frac{1}{2}\left(\nabla_{j} v_{i}-\nabla_{i} v_{j}\right)$.

\section{NUMERICAL SIMULATION RESULTS}

The results of numerical simulation [4] were obtained for the interaction of cylindrical steel projectile and a target made from 2024 aluminum alloy.

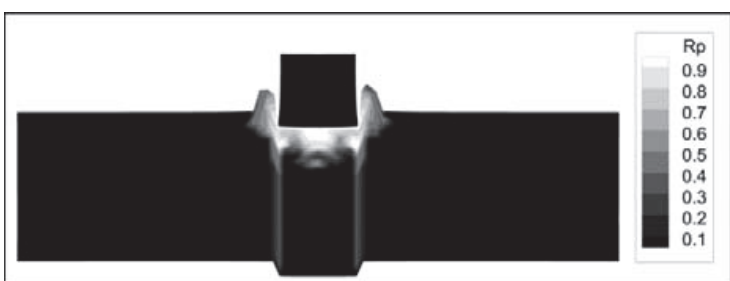

(a)

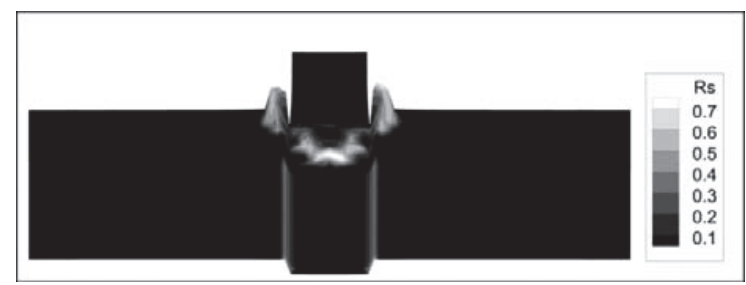

(b)

FIGURE 3. Material fracture zone during the compression (a) and tension (b) in the target material at the moment of $40 \mu \mathrm{s}$ $\left(V_{0}=200 \mathrm{~m} / \mathrm{s}\right)$. Considering all ultimate strains 


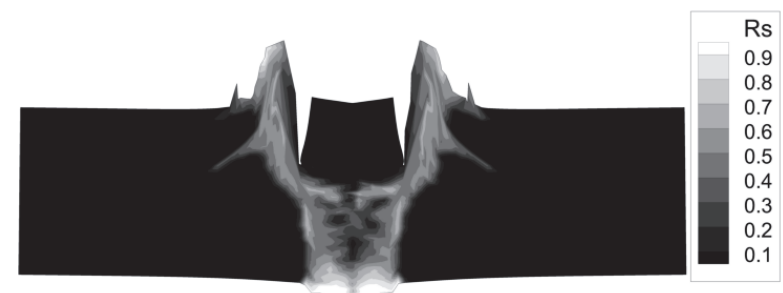

FIGURE 4. Material fracture zone during the tension in the target material at the moment of $40 \mu \mathrm{s}\left(V_{0}=600 \mathrm{~m} / \mathrm{s}\right)$

Elastic, plastic and strength characteristics of the materials were taken from work [5]. The projectile velocity was 200 and $600 \mathrm{~m} / \mathrm{s}$. Rs and Rp are volume ratios of the material fractured under tension and compression, respectively. The calculations yielded the following results.

Since the differences of ultimate elongation strains can reach 10 times for different symmetry directions, we have determined the contribution of each of the 6 strains into the resulting target fracture pattern.

If simulation does not contain all 6 conditions of the failure criterion, it will enable the determination of contribution of each of 6 ultimate strains into the resulting fracture pattern. The failure criterion consists of 6 independent relations. Varying each of the 6 ultimate plastic elongation and shear strains, and considering only a part of them allows determining the target zones with the fractures conditioned by the included relations.

For the case of failure criterion including all 6 correlations, the fracture zones in the target cross section are shown in Fig. 3 (the projectile initial velocity is $200 \mathrm{~m} / \mathrm{s}$ ). If $\varepsilon_{x x}^{\mathrm{p}}=0.07, \varepsilon_{y y}^{\mathrm{p}}=\varepsilon_{z z}^{\mathrm{p}}=0.21, \varepsilon_{y x}^{\mathrm{p}}=\varepsilon_{z x}^{\mathrm{p}}=0.035$ and $\varepsilon_{y z}^{\mathrm{p}}=0.105$, then the accounting all 6 fracture conditions leads to the target fracture in the form of shear plugging.

For the case of the failure criterion including only one relation $\varepsilon_{x x}^{\mathrm{p}}=0.07$, the fracture zones in the target cross section are shown in Fig. 2. In this case there is no shear plugging, and the tension and compression fracture zones are concentrated immediately in the target zone around the projectile.

If the initial velocity of the projectile is increased to $600 \mathrm{~m} / \mathrm{s}$ then the failure criterion allows simulating not only shear plugging but also the formation of a crater (Fig. 4).

\section{SUMMARY}

Numerical simulation was performed for transtropic 2024 aluminum alloy under impact loading. The results demonstrate the importance of using various failure criteria for problems in 3D formulation.

The work was performed within the framework of the fundamental scientific researches of the state academies of sciences for 2013-2020.

\section{REFERENCES}

1. S. W. Tsai and E. M. Wu, J. Compos. Mater. 5, 58 (1971).

2. E. M. Wu, J. Compos. Mater. 6, 473 (1972).

3. L. I. Sedov, Continuum Mechanics (Nauka, Moscow, 1976).

4. G. R. Johnson, J. Appl. Mech., March, 95 (1977).

5. P. G. Miklyaev and L. B. Fridman, Anisotropy of Mechanical Properties of Materials (Metallurgy, Moscow, 1986). 